\title{
PAIR PRODUCTION IN AGN
}

\author{
C. Done and A. C. Fabian \\ Institute of Astronomy \\ Madingley Road \\ Cambridge CB3 0HA \\ U.K.
}

The X-ray luminosity and variability of many AGN are sufficiently extreme that any hard $\gamma$-rays produced in the source will collide with the X-rays and create electron-positron pairs, rather than escape. A small region where vast amounts of energy are produced, such as an AGN, is an ideal place to accelerate particles to relativistic energies and so produce $\gamma$-rays by Compton scattering. The observed X-ray spectra of AGN are hard and indicate that most of the luminosity is at the highest energies so that absorption of the $\gamma$-rays represents a large fraction of the energy flux, which can then be re-radiated at lower energies. Pairs can thus effectively reprocess much of the radiant power in an AGN.

Following on from the work on this process by Guilbert, Fabian \& Rees (1983), Fabian et al. (1986), Svensson (1987) and Zdziarski \& Lightman (1987), we have begun to study the range of parameter space that can lead to the observed spectra through pair-reprocessing. It is not a simple matter of taking the observed spectra and variability since these are what emerge after the $\gamma$-rays have been absorbed to form pairs which have re-radiated and scattered X-rays. The whole spectrum and its variability are changed by the pair plasma.

The luminosity-to-size ratio of an emission region, $L / R$, or its dimensionless counterpart, $\ell=L \sigma_{T} / R m_{e} c^{2}$, known as the compactness parameter, measures the probability that $\gamma$-rays are absorbed through X-ray collisions. Here we report on results obtained (Done \& Fabian 1988) for a range of values of $\ell$, a monoenergetic relativistic electron injection of $\gamma=10^{3}$ and a soft EUV photon blackbody. This represents the 'soft excess' now commonly found in many AGN, probably due to the disc, and the value of $\gamma$ was chosen so that the first-order inverse Compton spectrum extends to $\gamma$-ray energies. The spectrum is evolved in a time-dependent way following an improved version of the code described in Fabian et al. (1986). Inverse Compton scattering, pair production and annihilation and Comptonization by thermal pairs are all included. A selection of typical spectra are shown in Fig. 1.

The model is well able to reproduce the X-ray spectral power-law of energy index 0.6 - 0.8 reported by Mushotzky (1982) and Turner \& Pounds (1988). In most cases, however, it gives too many $\gamma$-rays to be commonly applicable and be consistent with the $\gamma$-ray background. It also produces too large an annihilation feature. It may nevertheless apply to some sources.

The variability of the source is also affected by pair reprocessing. Changes in $\ell$ can be amplified by radiation by the pairs, the numbers of which will be variable, and damped by the scattering in the thermal pairs and the way in which the pairs buffer the energy flow. 


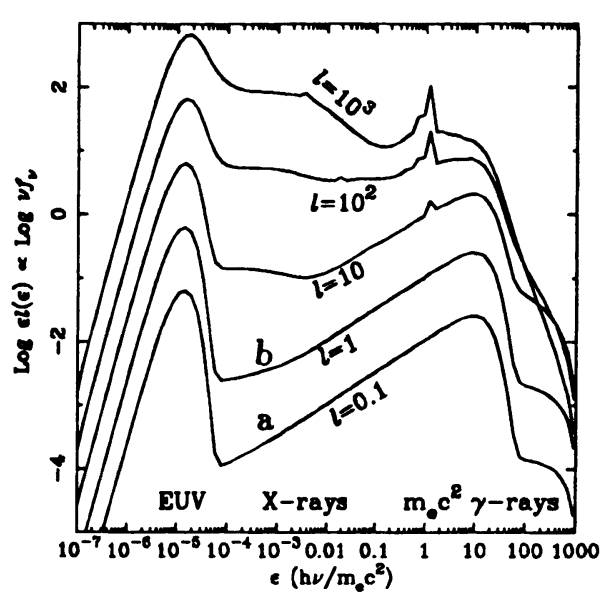

Figure 1. Calculated spectra $\ell=0.1-10^{3}$. Figure 2. Power spectra for the light curves

The light curves for Fig. 2 were taken from at $a$ and $b$ for the models with $\ell=0.1$ and 1 points $a$ and $b$.

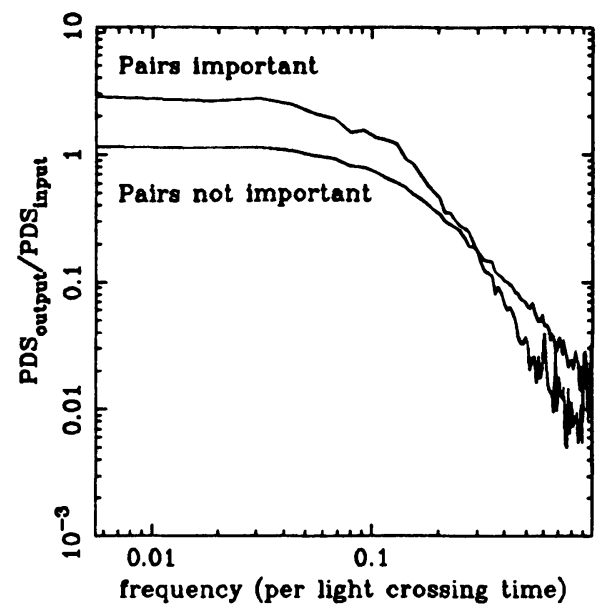
normalised to the power spectrum of the input.

We have studied these effects by varying the input power at random according to a white noise spectrum (up to the light-crossing frequency) and measuring the correlations and power spectrum of the emergent flux as a function of energy. Dividing the power spectrum of the output by that of the input gives the frequency response of the system as shown in Fig. 2. Where the spectrum is formed by pair reprocessing the power is damped at high frequency and amplified at low frequencies. The high frequency damping means that regular large-amplitude variability in such sources can only occur on about 10 light crossing times. This means that any simple of determinations of $\ell$ in a source based on its variability are likely to be underestimated.

We are proceeding to investigate a broader region of parameter space in which the acceleration mechanism also acts on the pairs and in which higher order inverse Comptonscattering are important.

Gabriele Ghisellini is thanked for many helpful discussions. CD and ACF thank the Carnegie Trust and the Royal Society, respectively.

\section{REFERENCES}

Done, C., and Fabian, A.C. 1988, preprint.

Fabian, A.C., Blandford, R.D., Guilbert, P.W., Phinney, E.S., and Cuellar, L. 1986, M.N.R.A.S., 221, 931.

Guilbert, P.W., Fabian, A.C., and Rees, M.J. 1983, M.N.R.A.S., 205, 593.

Lightman, A.P., and Zdziarski, A.A. 1987, Ap. J., 319, 643.

Mushotzky, R.F. 1982, Ap.J., 256, 92.

Svensson, R. 1987, M.N.R.A.S., 227, 403.

Turner, T.J., and Pounds, K.A. 1988, preprint. 


\section{DISCUSSION}

KAZANAS You have shown us the effects of the pairs in the time-variability power spectrum. I would like to point out that the difficulty in accounting for the observed spectra is related to the large amount of power observed in the low rather than high frequencies.

DONE The whole question of variability is not well understood. At low frequencies the power spectrum rises, but there is still power at high frequencies that needs to be explained.

LAWRENCE Do you happen to know what the compactness parameter would be for the fireball in an atomic bomb? I wonder if we have a good laboratory here to study quasars!

DONE Actually no, atomic bombs are much less compact than AGNs. If 1 gram of matter is converted to energy in 1 second, and if the initial fireball has a radius of 1 meter, then the compactness $\ell \sim 10^{-9}$. So even if all the energy of the bomb was emitted in the $X$-ray $/ \gamma$-ray region (which it isn't) pairs wouldn't be important. 